\title{
A New Centrality Measure for Social Network Analysis Applicable to Bibliometric and Webometric Data
}

\author{
HILDRUN KRETSCHMER ${ }^{1,2,3}$, \\ THEO KRETSCHMER ${ }^{3}$ \\ ${ }^{1}$ Department of Library and Information Science, Humboldt-University Berlin, \\ Dorotheenstr. 26, D-10117 Berlin, Germany \\ ${ }^{2}$ The School of Humanities and Social Sciences, Dalian University of Technology, Dalian, 116023, China \\ ${ }^{3}$ COLLNET Center, Hohen Neuendorf, Germany
}

\section{Abstract}

In the literature there are a large number of publications in sociology, in computer science or in information sciences, as well as in studies of collaboration in science describing the studies of social networks with unweighted ties because measures involving unweighted ties are easier to calculate. It is not surprising that there are few studies on networks with weighted ties since they not only need more complex formulas but need a process of quantification when quantitative empirical data are not directly available.

However quantitative empirical data are directly available under the condition of using bibliometric or webometric data.

In conclusion new complex measures of the degree centrality are introduced including weighted ties possible for use of the analysis of co-authorship or citation networks. Both co-authorship relations and citations are well quantified data (weighted ties).

These new measures are applied to a co-authorship network as an example.

\section{Introduction}

There is a rapid increase of network analysis in several scientific disciplines beginning some decades ago. The social network analysis (SNA) is developed especially in sociology and in social psychology in collaboration with mathematics, statistics and computer science.

Otte and Rousseau [1] showed that social network analysis (SNA) can also be used successfully in the information sciences, as well as in studies of collaboration in science. The authors showed interesting results by the way of an example of the co-authorship network of those scientists who work in the area of social network analysis.

A social network is a set of nodes (social actors) connected by a set of ties. The ties between the nodes can be directed or undirected and weighted or unweighted.

SNA is used to extract patterns of relationships between social actors in order to discover the underlying social structure. Various measures are offered by network analysis. The most used measures are density of the network and the centrality measures: degree centrality, betweenness and closeness.

This paper is focused on degree centrality. 
Coulon has pointed out [2] in his literature review about the use of social network analysis there is a large number of publications describing the studies of networks with unweighted ties because measures involving unweighted ties are easier to calculate. According to Coulon's opinion it is not surprising that there are few studies on networks with weighted ties since they not only need more complex formulas but need a process of quantification when quantitative empirical data are not directly available.

However quantitative empirical data are directly available under the condition of using bibliometric or webometric data.

In conclusion a new complex measure of degree centrality is introduced including weighted

ties suitable for analyzing co-authorship-, citation- or Web networks. Co-authorship relations, citations, Web visibility rates or hyperlinks are well quantified data (weighted ties).

In this paper the new measure is applied to a co-authorship network as an example.

\section{Presentation of the Original Measure for Degree Centrality}

The nodes of a social network can be individuals, teams, institutions, etc. The relationships (ties) between the nodes can also be of many kinds for example, friendship, business, economic, etc. In this paper we are looking at scientists as nodes and at co-authorship relations, citations or hyperlinks as ties.

The original used measures of social network analysis (SNA) are related to Wassermann \& Faust [3]:

- The Degree Centrality $\left(\mathrm{DC}_{\mathrm{A}}\right)$ of a node $\mathrm{A}$ is equal to the number of nodes (or ties) to which this node is connected.

For example, in collaboration networks in science the Degree Centrality of a node A is equal to the number of his/her collaborators or co-authors. An actor (node) with a high degree centrality is active in collaboration. He/she has collaborated with many scientists.

- In correspondence with Wassermann and Faust the Group Degree Centralization quantifies the variability or dispersion of the individual Degree Centralities of the nodes. Centralization describes the extent to which the links (ties) are organized around particular focal nodes, i.e. it provides a measure on the extent to which a whole network has a centralized structure.

There are several degree based measures of graph centralization. One of them is as follows:

$$
\Sigma_{\mathrm{i}=1}^{\mathrm{v}}\left(\mathrm{DC}_{\mathrm{L}}-\mathrm{DC}_{\mathrm{i}}\right)
$$

$\mathrm{GDC}=$

$$
(\mathrm{v}-1)(\mathrm{v}-2)
$$

The $\mathrm{DC}_{\mathrm{i}}$ in the numerator are the $\mathrm{v}$ Degree Centralities of the nodes and $\mathrm{DC}_{\mathrm{L}}$ is the largest observed value.

This index reaches its maximum value of 1 when one actor (node) has collaborated with all other $\mathrm{v}-1$ actor, and the other actors interact only with this one, central actor. This is exactly the case in a star graph. The index attains its minimum value of 0 when all degrees are equal.

\section{Comparison of Weighted and Unweighted Degree Centrality Measures Explained on the Basis of Co-authorship Networks}

\subsection{General Remarks}

Using the unweighted measure means the ties (or nodes) are counted independently from the strength of the ties. 
However in analyzing bibliometric or webometric networks several sorts of methods are developed to measure the strength of a tie between a pair of nodes A and B.

Application of similarity coefficients is one of them. While many similarity coefficients were proposed in various research fields, Salton's measure or Jaccard index were most used in

scientometric studies (Glänzel [4] , Miquel and Y. Okubo [5], Katz [6] ). Zitt et al. [7] introduced a probabilistic indicator to measure strength of scientific linkages between partners. Yamashita and Okubo [8] have presented a new probabilistic partnership index (PPI).

However in this paper we don't yet consider the above mentioned kinds of measuring the strength of ties. Compared with this we are looking for a co-authorship relation or for a citation, etc., as the basic unit of links:

- $\quad$ The strength of a tie between a pair of nodes $\boldsymbol{A}$ and $\boldsymbol{B}_{\boldsymbol{i}}$ can be measured by the number of basic units which exists between $\mathrm{A}$ and $\mathrm{B}_{\mathrm{i}}$ :

$$
\mathrm{U}_{\mathrm{ABi}}
$$

- $\quad$ The total strength of all of the ties between a node $A$ and all of the nodes

$B_{i}(i=1,2 \ldots z)$ to which this node $A$ is connected is equal to the sum of the strengths of these ties:

$$
\mathrm{TR}_{\mathrm{A}}=\Sigma_{\mathrm{i}} \mathrm{U}_{\mathrm{ABi}}
$$

- $\quad$ The total strength $T$ of all of the ties in a network with v nodes $X_{j}$ is equal to the total sum of $\mathrm{TR}_{\mathrm{Xj}}$ divided by 2 :

$$
\mathrm{T}=\left(\Sigma_{\mathrm{j}=1}^{\mathrm{v}} \mathrm{TR}_{\mathrm{Xj}}\right) / 2
$$

Let us compare weighted and unweighted degree centrality measures under the following conditions:

- First condition: $\mathrm{DC}_{\mathrm{A}}=$ const, $\mathrm{TR}_{\mathrm{A}}$ is changing, $\mathrm{U}_{\mathrm{AB} 1}=\mathrm{U}_{\mathrm{AB} 2},=\ldots=\mathrm{U}_{\mathrm{ABz}}$

- Second condition: $\mathrm{DC}_{\mathrm{A}}$ is changing, $\mathrm{TR}_{\mathrm{A}}=$ constant, $\mathrm{U}_{\mathrm{AB} 1}=\mathrm{U}_{\mathrm{AB} 2},=\ldots=\mathrm{U}_{\mathrm{ABz}}$

- Third condition: $\mathrm{DC}_{\mathrm{A}}=$ constant, $\mathrm{TR}_{\mathrm{A}}=$ constant, $\mathrm{U}_{\mathrm{ABi}} \leq \mathrm{U}_{\mathrm{ABj}}$ or $\mathrm{U}_{\mathrm{ABi}} \geq \mathrm{U}_{\mathrm{ABj}}$

\subsection{First Condition}

Regarding the variation of the strengths of the ties let us have a view at the following example:

Using the unweighted measure of the degree centrality says the degree centralities of the scientists $\mathrm{E}$ and $\mathrm{F}$ are equal in both networks (Fig. 1, left side and right side) although on the right side the strengths of the scientist E's ties are several times higher than on the left.
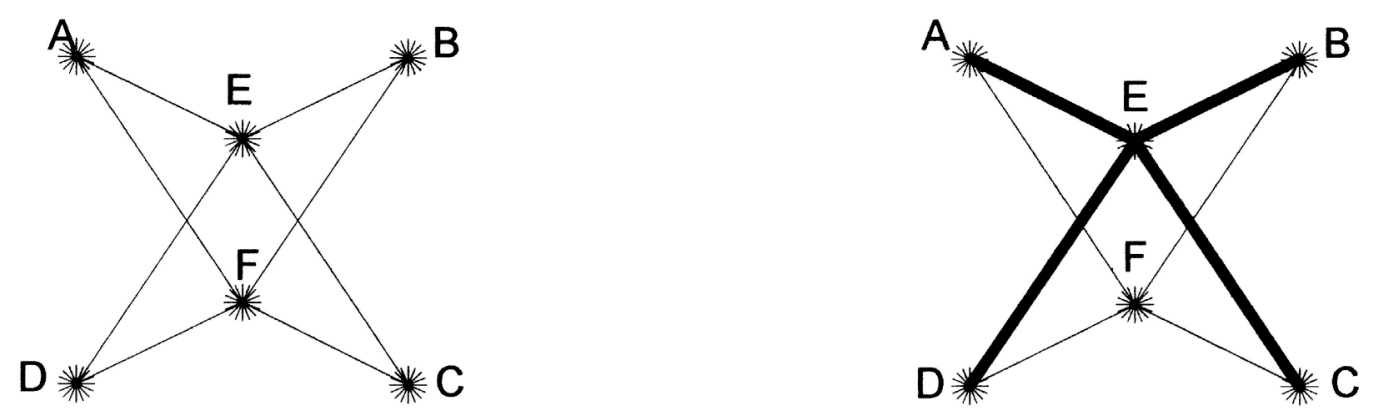

Fig. 1: Comparison between two networks First condition: $D C_{E}=$ const, $T R_{E}$ is changing, $U_{E A}=U_{E B}=U_{E C}=U_{E D}$

What does it mean in co-authorship networks? At first glance scientist $\mathrm{E}$ is more centralized in the right side network than in the other network. Additionally, let us take into consideration the theoretical background. 
Co-authored research papers are assumed to signal research cooperation and associated knowledge flows and exchanges Calero, van Leeuven \& Tijssen [9] . In continuation we assume the knowledge flow between a pair of collaborators A and B is increasing with increasing number of co-authorship relations (strength of the tie). The number of co-authorship relations between a pair of nodes $\mathrm{A}$ and $\mathrm{B}$ is equal to the number of their joint multi-authored papers.

Analogous considerations can be made in citation or Web networks.

Because of these considerations the centrality of a scientist A is increasing with both increasing number of collaborators (degree centrality) and increasing total number of co-authorship relations with these collaborators. This condition is not fulfilled using the original degree centrality.

Whereas citations or hyperlinks are well defined in our field there maybe differences regarding the term "co-authorship relation". Thus, explanation is necessary as follows:

Counting the total number of co-authorship relations $\left(T R_{A}\right)$ of an author $A$ :

Given one multi-authored paper $\mathrm{p}_{\mathrm{Ai}}$ of the scientist $\mathrm{A}$ with $\mathrm{m}_{\mathrm{Ai}}$ co-authors then the number of co-authorship relations of $\mathrm{A}$ is equal to $\mathrm{m}_{\mathrm{Ai}}$.

Let us assume the number of multi-authored papers of the scientist $\mathrm{A}$ is equal to $\mathrm{z}$. the total number of co-authorship relations of $A$ is equal to the sum of the relations of the $\mathrm{z}$ multi-authored papers:

$$
\mathrm{TR}_{\mathrm{A}}=\Sigma_{\mathrm{i}=1}^{\mathrm{Z}} \mathrm{m}_{\mathrm{Ai}}
$$

Following

Whereas the number of co-authorship relations between a pair $A$ and $B_{i}\left(U_{A B i}\right)$

is equal to the number of joint multi-authored papers the total number of co-authorship relations of the author $\mathrm{A}$ in collaboration with more than one co-author $\left(\mathrm{TR}_{\mathrm{A}}\right)$ can be either equal or higher than the total number of multi-authored papers.

\subsection{Second Condition}

In social networks usually the number of actors to which an actor A is connected can vary independently from the total strength of the ties.

For example in Fig. 1, the number of collaborators of $\mathrm{E}$ or $\mathrm{F}$ is constant but the total strength of the ties (total number of co-authorship relations) is different.

Vice versa, in another network the authors $\mathrm{G}$ and $\mathrm{J}$ can have the same total number of co-authorship relations but the number of collaborators is different. In Fig. 2 the total number of co-authorship relations of $\mathrm{G}$ (equal to 8 ) is spread out over 2 collaborators but the same total number of coauthorship relations of $\mathrm{J}$ (equal to 8) is spread out over 4 collaborators.
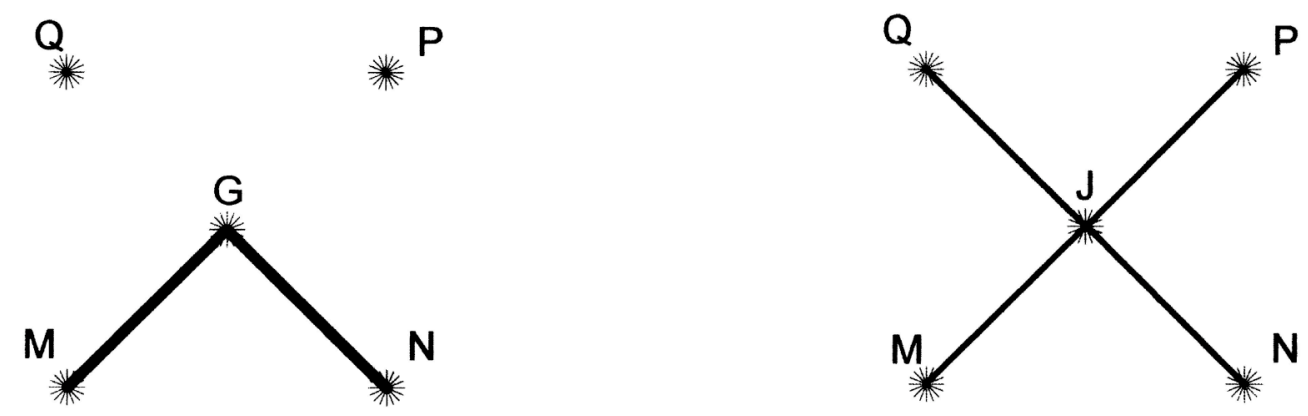

Fig. 2: Comparison between two networks

Second condition: $D C_{X}$ is changing, $T R_{X}=$ constant, $U_{X Y 1}=U_{X Y 2},=\ldots=U_{X Y z}$ with $X=G=J, Y_{i}$ are all of the nodes $Y_{i}(i=1,2 \ldots z)$ to which the special $X$ is connected 
General Stipulation for a weighted degree centrality measure of an actor:

If two variables can vary independently of each other, the following condition has to be fulfilled: If one variable remains constant and the other variable assumes a higher weighted degree centrality measure must assume a higher value. This requirement will be met by the geometric mean of the magnitudes of the two variables:

$A$ weighted degree centrality measure of a node $A$ is equal to the geometric mean of the number of nodes to which this node is connected and the total strength of the ties.

Whereas the original Degree Centralities of the scientists $\mathrm{E}$ and $\mathrm{F}$ are equal in both networks in Fig. 1 a weighted degree centrality measure of $\mathrm{E}$ is higher in the network on the right side of Fig. 1. However in Fig. 2 both the Degree Centrality and a weighted degree centrality measure of J are higher than both corresponding values of $\mathrm{G}$ because the total sum of co-authorship relations remains constant.

\subsection{Third Condition}

In the examples in Fig. 1 and in Fig. 2 we have counted the number of collaborators of a scientist A on the basis of equal strengths of the ties between the pairs of collaborators. However how to measure the number of collaborators on the basis of unequally weighted ties?
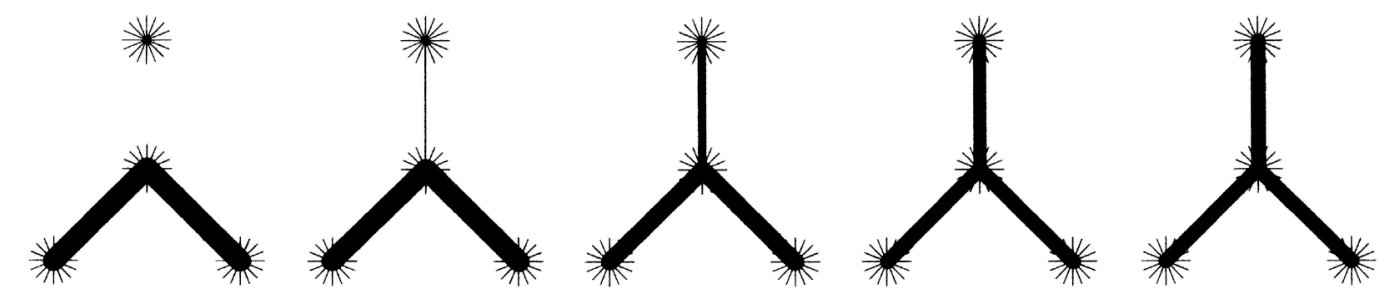

\section{Fig. 3: Comparison between five networks with four nodes per network and node $A$ in the middle of each of the networks}

$$
\text { Third condition: } D C_{A}=\text { constant, } T R_{A}=\text { constant, } U_{A B i} \leq U_{A B j} \text { or } U_{A B i} \geq U_{A B j}
$$

There are five networks in Fig. 3 with four nodes per network. The first network on the left side shows the number of collaborators of the scientist A (node in the middle of the four nodes) is equal to 2 but the last network on the right side says it is equal to 3. Moving our eyes from left to right at first glance we have the impression there is a continuous change of the "number" of collaborators from 2 to 3, i.e. in the middle networks the "number" of collaborators of A is between 2 and 3 .

In Fig. 3 is valid: The total sum of co-authorship relations remains constant however the spread over the (possible) collaborators is changing.

How to measure the "number" of collaborators under these kinds of conditions?

In Table 1 the total number of co-authorship relations $\left(\mathrm{TR}_{\mathrm{X}}\right)$ is equal for all of the scientists in the column (A, B, C or D) in co-authorship with the scientists in the row (E or F). However the strengths of the ties between the pairs of collaborators (values in the cells of the matrix) are different.

The number of collaborators of $\mathrm{A}$ : $\left(\mathrm{DC}_{\mathrm{A}}\right)$ is clearly 1 and the number of collaborators of $\mathrm{D}$ : $\left(\mathrm{DC}_{\mathrm{D}}\right)$ is also clearly 2 . However, if you ask B for the number of his collaborators, he will possibly give the answer „one” since the number of co-authorship relations between him and the collaborator $\mathrm{E}$ (equal to1) in relation to the number of co-authorship relations between him and collaborator $\mathrm{F}$ (equal to 99), is so small that this fact could be neglected. By contrast, C might say that the number of his collaborators is 2 because the co-authorship relations are almost equally distributed. 
Table 1: Unequally weighted ties between pairs of collaborators

\begin{tabular}{|l|l|l|l|l|l|}
\hline $\mathrm{X} / \mathrm{Y}$ & $\mathrm{E}$ & $\mathrm{F}$ & $\mathrm{TR}_{\mathrm{X}}$ & $\mathrm{DC}_{\mathrm{X}}$ & $2^{\mathrm{H}(\mathrm{Ki})}$ \\
\hline $\mathrm{A}$ & & 100 & 100 & 1 & 1 \\
\hline $\mathrm{B}$ & 1 & 99 & 100 & $>1 \approx 1$ & 1.06 \\
\hline $\mathrm{C}$ & 49 & 51 & 100 & $<2 \approx 2$ & 1.99 \\
\hline $\mathrm{D}$ & 50 & 50 & 100 & 2 & 2 \\
\hline
\end{tabular}

Note: The calculation of $2^{\mathrm{H}(\mathrm{Ki})}$ can be found below.

\section{Conclusions:}

The idea is to search for a function that upon an equal distribution of weights on the elements to be counted is equal to the "number" of elements. The greater the deviation is from this equal distribution, the smaller shall be the value of the function.

In the example the elements to be counted were the collaborators $\mathrm{E}$ or $\mathrm{F}$ and the weights (or strength of ties) were the number of co-authorship relations existing between the corresponding scientists A, B, $\mathrm{C}$, or $\mathrm{D}$ and each of these collaborators.

According to the definition for the sought-after function the ,number” of the collaborators of $\mathrm{C}$ is to be smaller than 2 but almost 2. The „number” of collaborators of B is to be greater than 1 , but almost 1 . A function that meets those requirements is the transformed entropy $2^{\mathrm{H}}$. The results are shown in Table 1. The calculation is shown below.

Calculation of the entropy $H\left(\mathrm{~K}_{\mathrm{i}}\right)$ :

There is a series of numbers $\mathrm{K}_{\mathrm{i}}(\mathrm{i}=1,2, \ldots \mathrm{z}), \mathrm{K}_{\mathrm{i}} \neq 0$

$\mathrm{h}_{\mathrm{i}}=\mathrm{K}_{\mathrm{i}} / \Sigma_{\mathrm{i}=1}^{\mathrm{z}} \mathrm{K}_{\mathrm{i}}$

$\mathrm{H}\left(\mathrm{K}_{\mathrm{i}}\right)=-\Sigma_{\mathrm{i}=1}^{\mathrm{z}} \mathrm{h}_{\mathrm{i}} \cdot \lg _{2} \mathrm{~h}_{\mathrm{i}}$

Stipulation: If $\Sigma_{\mathrm{i}=1}^{\mathrm{z}} \mathrm{K}_{\mathrm{i}}=0$ then $2^{\mathrm{H}(\mathrm{Ki})}=0$

In calculation of the "number" of collaborators of the scientist $A$ the term $\mathrm{K}_{\mathrm{i}}$ is equal to the strength of a tie between a pair of the nodes $A$ and $B_{i}$ :

$$
\mathrm{K}_{\mathrm{i}}=\mathrm{U}_{\mathrm{ABi}}
$$

The "number" of collaborators of the scientist $\mathrm{A}$ is equal to $2^{\mathrm{H}(\mathrm{Ki})} \quad$ :

$$
\text { "DC" }{ }_{\mathrm{A}}=2^{\mathrm{H}(\mathrm{Ki})}
$$

\section{The Complex Measure of Degree Centrality $\left(C D C_{A}\right)$ of a Node $A$}

The considerations in paragraph 3 are resulting in the following definition for the Complex Degree Centrality $\mathrm{CDC}_{\mathrm{A}}$ measure of a node A:

The Complex Measure of Degree Centrality $C D C_{A}$ of a node $A$ is equal to the geometric mean of the "number" of nodes to which this node is connected and the total strength of the ties.

Therefore, for the analysis of networks based on co-authorship data the Complex Degree Centrality of a scientist $\mathrm{A}$ is defined as:

$\mathrm{CDC}_{\mathrm{A}}=$ ("Number" of collaborators of scientist A · Total sum of co-authorship relations of the scientist $\mathrm{A})^{1 / 2}$

$\mathrm{CDC}_{\mathrm{A}}=\left(\text { " } \mathrm{DC} "{ }_{\mathrm{A}} \cdot \mathrm{TR}_{\mathrm{A}}\right)^{1 / 2}$ 
As mentioned above Group Degree Centralization quantifies the variability or dispersion of the individual Degree Centralities of the nodes. One of the several degree based measures of graph centralization was described in formula 1.

The Complex Centralization describes the extent to which the links (ties) are organized around both particular focal nodes and ties, i.e. it provides a measure on the extent to which a whole network has a centralized structure.

Using the Complex Degree Centrality a new Complex Group Degree Centralization measure is proposed describing the dispersion in a connected graph, i.e., there is a "path" between each pair of nodes $\mathrm{X}_{\mathrm{i}}$ and $\mathrm{X}_{\mathrm{j}}$ :

$$
\begin{aligned}
& \mathrm{GCDC}=1-\left(\mathrm{H}\left(\mathrm{CDC}_{\mathrm{Xi}}\right)-\mathrm{H}_{\min }\right) /\left(\mathrm{H}_{\max }-\mathrm{H}_{\min }\right) \\
& \text { with } \mathrm{H}_{\max }=\lg _{2} \mathrm{~V}
\end{aligned}
$$

Stipulation for calculation of the minimum Entropy $\mathrm{H}_{\min }$ :

Given a network with $\mathrm{v}$ nodes $\mathrm{X}_{\mathrm{i}}$ and with the total strength $\mathrm{T}$ of all of the ties in this network:

- The total strength of all of the ties between the node $\mathrm{X}_{1}$ and all of the other nodes should be equal to $\mathrm{T}$ :

$$
\mathrm{TR}_{\mathrm{X} 1}=\mathrm{T}
$$

- The strength of the tie between $X_{1}$ and $X_{2}$ should be equal to T-(v-2):

$$
\mathrm{U}_{\mathrm{X} 1 \mathrm{X} 2}=\mathrm{T}-(\mathrm{v}-2)
$$

- The strength of a tie between $X_{1}$ and $X_{i}$ with $i(i=3,4, . . v)$ is equal to 1 :

$$
\mathrm{U}_{\mathrm{X} 1, \mathrm{Xi}}=1
$$

GCDC reaches its maximum value of 1 when one actor (node) has collaborated with all other v-1 actors, and the other actors interact only with this one, central actor and when there is a maximum strength of one of the ties. This is exactly the case in a star graph with a maximum strength of the tie between the central node and one of the other nodes. The index attains its minimum value of 0 when all Complex Degree Centrality degrees are equal.

The Complex Measure of Degree Centrality is applied to a co-authorship network as an example. The new measure is compared with the original.

\section{Data}

COLLNET is a global interdisciplinary research network under the title: "Collaboration in science and in technology" (www.collnet.de). In a former study (Kretschmer \& Aguillo [10] ) a request was made to all the 64 COLLNET members for their complete bibliographies, independently of the type of the publications and independently from the date of appearance of these publications.

From these bibliographies all publications were selected that appeared in co-authorship between at least two COLLNET members. Thus, it concerns 223 bibliographic multi-authored publications. From this, the respective number of common publications between two members was determined as the basis for the analysis of the co-authorship network.

The co-authorship network developed according to this method covers the entire lifetime collaboration between the COLLNET members.

The last COLLNET data are from June 2003. These data are used in this paper, too.

\section{Results and Conclusion}

The structure of the co-authorship network obtained from the bibliographies is shown in Fig. 4 with unweighted ties. 


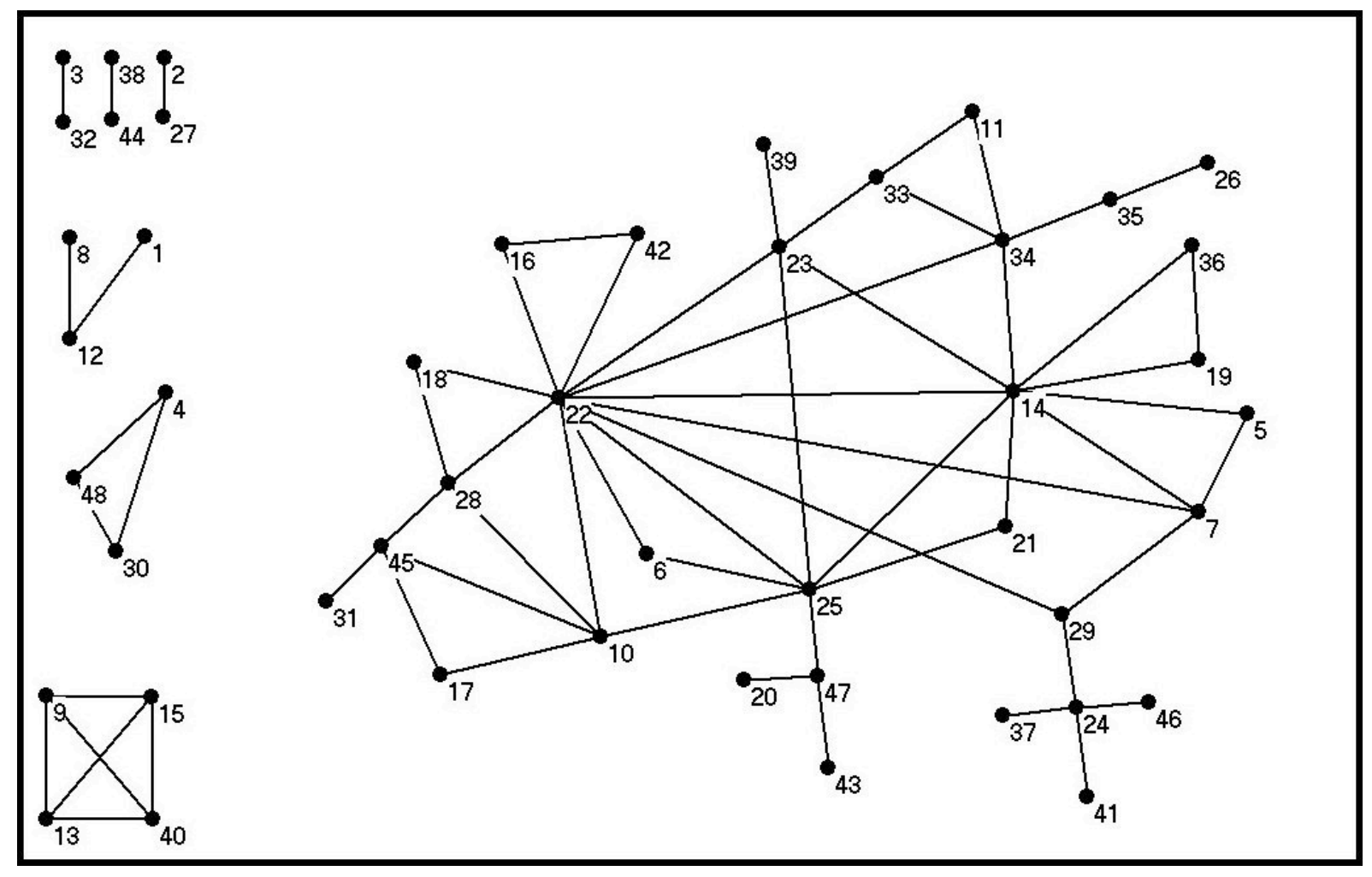

Fig. 4: Co-authorship network of the COLLNET members (Kretschmer \& Aguillo, 2004)

Legend (Fig. 4):
1. Isidro Aguillo
2. Petra Ahrweiler
3. R. Ambuja
4. Bassecoulard
5. Aparna Basu
6. Donald deB. Beaver
7. Sujit Bhattacharya
8. Maria Bordons
9. Martina Brandt
10. Mari Davis
11. Leo C.J. Egghe
12. Isabel Gomez
13. Ulla Grosse
14. Brij Mohan Gupta
15. Frank Hartmann
16. Frank Havemann
17. William W. Hood
18. Margriet Jansz
19. Karisiddappa 20. Sylvan Katz
21. Ved P. Kharbanda
22. Hildrun Kretschmer
23. Ramesh Kundra
24. Loet Leydesdorff
25. Liming Liang
26. Sofía Liberman
27. Zeyuan Liu
28. Valentina Markusova
29. Martin Meyer
30. Yoshiko Okubo
31. Farideh Osareh
32. Koti S. Raghavan
33. Ravichandra Rao
34. Ronald Rousseau
35. Jane Russell
36. Shivappa Sangam
37. Andrea Scharnhorst
38. Annedore Schulze
39. Dimiter Tomov
40. Rainer Voss
42. R. Wagner-Döbler
43. Yan Wang
44. Vera Wenzel

46. Paul Wouters 47. Yishan Wu

48. Michel Zitt

45. C. S. Wilson

\section{Paul Wouters 47. Yishan Wu}

49.-64. are singletons up to June 2003. The 16 singletons are not included in the figure.

For demonstration of the differences between the original and the complex measures of degree centrality we have extracted two scientists with the same Degree Centrality from Fig. 4: $\mathrm{DC}_{24}=\mathrm{DC}_{45}=4$. The node 24 can be found in the middle of the left network of Fig. 5 and the node 45 in the middle of the right network.

The strengths of the ties of the node 24 are as follows:

$\mathrm{U}_{24 ; 29}=1, \mathrm{U}_{24 ; 37}=1, \mathrm{U}_{24 ; 41}=2, \mathrm{U}_{24 ; 46}=5$ with the total strength $\mathrm{TR}_{24}=9$

The strengths of the ties of the node 45 are:

$\mathrm{U}_{45 ; 28}=5, \mathrm{U}_{45 ; 31}=6, \mathrm{U}_{45 ; 17}=20, \mathrm{U}_{45 ; 10}=10$ with the total strength $\mathrm{TR}_{45}=41$ 
The "number" of collaborators "DC" ${ }_{24}=3.155$ is rather similar as the "number" of collaborators "DC" ${ }_{45}=3.429$.

However the Complex Degree Centrality of node 45 is higher than the Complex Degree Centrality of node 24 because of the higher total strength:

$$
\begin{aligned}
& \mathrm{CDC}_{24}=\left(" \mathrm{CDC}{ }_{\mathrm{A}} \cdot 9\right)^{1 / 2}=(3.155 \cdot 9)^{1 / 2}=\mathbf{5 . 3 3} \\
& \mathrm{CDC}_{45}=\left(" \mathrm{CDC}{ }_{\mathrm{A}} \cdot 41\right)^{1 / 2}=(3.43 \cdot 41)^{1 / 2}=\mathbf{1 1 . 8 6}
\end{aligned}
$$
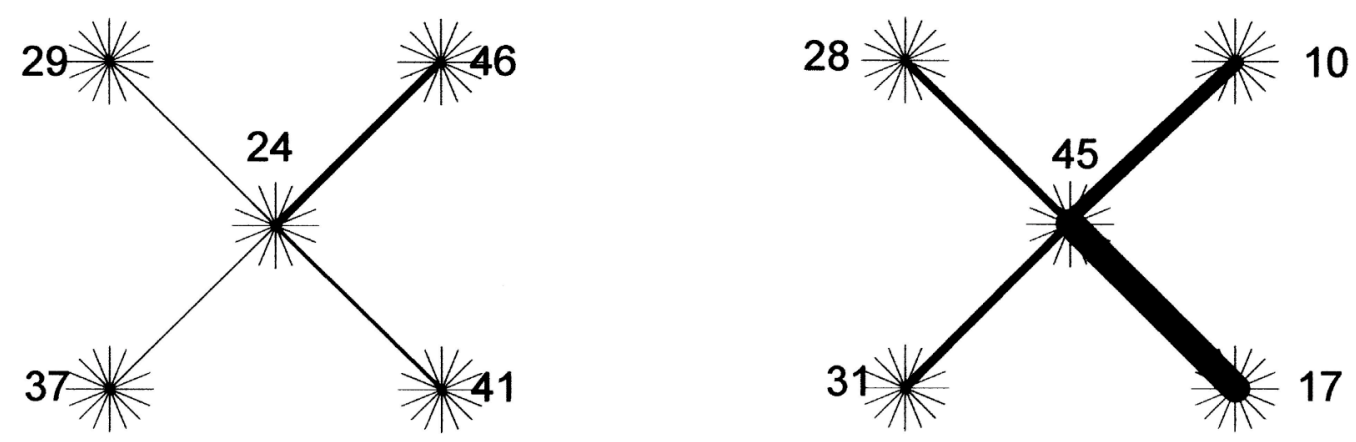

Fig. 5: Comparison between two scientists with equal Degree Centrality

For another comparison we have selected 3 triads for calculation of the degree centralities of the nodes and the group degree centralization (Fig. 6). The original centrality measures say the degree centralities are equal for all of the nodes and the group degree centralization is equal for all of the three triads.

At first glance the centralization of the right triad is higher (with node 48 in the center) than the centralization of the other triads. Indeed the Complex Degree Centrality of the node 48 is higher than the original measure and the same with the Complex Group Degree Centralization. In detail:

The strengths of the ties are as follows:

- left triad: $\mathrm{U}_{5 ; 14}=\mathrm{U}_{14 ; 7}=\mathrm{U}_{5 ; 7}=1$

- triad in the middle: $\mathrm{U}_{15 ; 9}=5, \mathrm{U}_{9 ; 40}=5, \mathrm{U}_{40 ; 15}=4$,

- right triad: $\mathrm{U}_{48 ; 30}=5, \mathrm{U}_{30 ; 4}=2, \mathrm{U}_{4 ; 28}=28$

The Complex Degree Centralities of the nodes are:

- left triad: $\mathrm{CDC}_{5}=2, \mathrm{CDC}_{7}=2, \mathrm{CDC}_{14}=2$

with the Complex Group Degree Centralization $\quad$ GCDC $=0$

- triad in the middle: $\mathrm{CDC}_{15}=4.23, \mathrm{CDC}_{9}=4.47, \mathrm{CDC}_{40}=4.23$

with the Complex Group Degree Centralization GCDC $=\mathbf{0 . 0 0 2 5 5}$

- right triad: $\mathrm{CDC}_{48}=7.11, \mathrm{CDC}_{30}=3.567, \mathrm{CDC}_{4}=6.19$

with the Complex Group Degree Centralization GCDC $=0.20027$
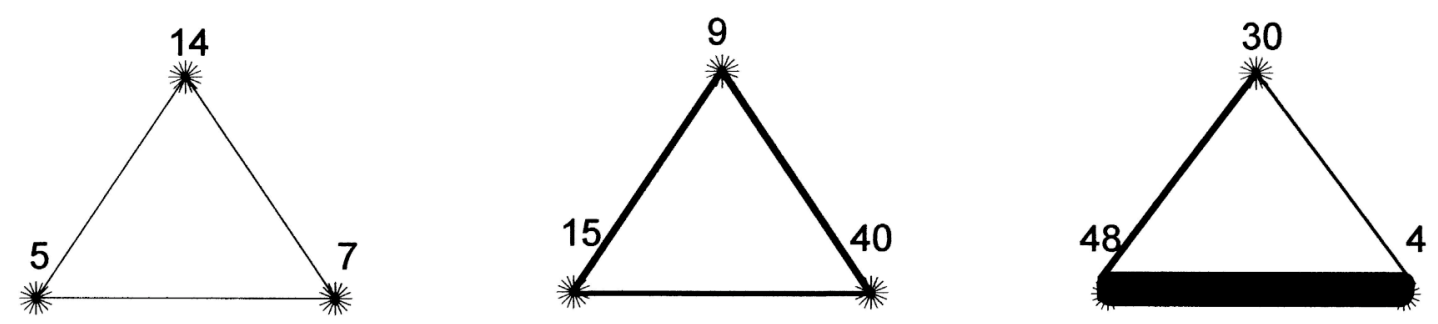

Fig. 6: Selection of three triads 
After demonstration of these new introduced Complex Degree Centrality Measures in this pilot study this new method should be empirically tested in future analyses of bibliographic citation or coauthorship networks as well as in analyses of Web networks.

\section{References}

1. E. Otte \& R. Rousseau, Social network analysis: a powerful strategy, also for the information sciences. Journal of Information Science, 28, 443-455, 2002

2. F. Coulon, The use of social network analysis in innovation research: a literature revie, 2005.

http://www.druid.dk/conferences/winter2005/papers/dw2005-305.pdf (The Web search took place on January 1, 2006)

3. S. Wasserman \& K. Faust, Social network analysis. Methods and applications.

Cambridge: Cambridge University Press, 1994

4. W. Glänzel \& A. Schubert, Analyzing scientific networks through co-authorship. In H.F. Moed et. al. (Eds.), Handbook of QuantitativeScience and Technology Research, (pp.257-276), The Netherlands: Kluwer Academic Publishers, 2004

5. J.F. Miquel, \& Y. Okubo, Structure of International Collaboration in Science-Part II: Comparisons of Profiles in Countries using a Link Indicator, Scientometrics 29. No.2, 271-297, 1994

6. J.S. Katz, Geographical proximity and scientific collaboration, Scientometrics 31, 31-34, 1994

7. M. Zitt, E. Bassecoulard \& Y. Okubo, Shadows of the Past in International Cooperation: Collaboration profiles of the top five producers of science, Scientometrics. 47, No. 3, 627-657, 2000

8. Yamashita \& Y. Okubo, Patterns of scientific collaboration between Japan and France: Inter-sectoral analysis using probabilistic Partnership Index (PPI). In: Peter Ingwersen \& Birger Larsen (Eds.). Proceedings of the $10^{\text {th }}$ ISSI International Conference on Scientometrics and Informetrics, July 24-28, 2005, Stockholm, Sweden, Volume 2. Published by Karolinska University Press: Stockholm, 2005, 517526,2005

9. C. Calero, T.N. van Leeuven \& J.W. Tijssen (2005). Reseaarch networks of pharmaceutical firms: geographiccal patterns of research collaboration within and between firms. In: Peter Ingwersen \& Birger Larsen (Eds.). Proceedings of the $10^{\text {th }}$ ISSI International Conference on Scientometrics and Informetrics, July 24-28, 2005, Stockholm, Sweden, Volume 1. Published by Karolinska University Press: Stockholm, 2005, 310-315, 2005

10. Kretschmer \& I. Aguillo, Visibility of collaboration on the Web. Scientometrics. Vol. 61, No. 3, 405-426, 2004 